\title{
Comparison between the Effectiveness of Manual Acupuncture and Laser Acupuncture at EX-B3 Weiwanxiashu in a Rat Model of Type 2 Diabetes
}

\author{
Ahmad Aulia Jusuf', Yoshua Viventius ${ }^{2,3}$, Wahyuningsih Djaali ${ }^{2,4, *}$ \\ ${ }^{1}$ Department of Histology, Faculty of Medicine, Universitas Indonesia, Jakarta, Indonesia \\ ${ }_{2}^{2}$ Medical Acupuncture Specialist Program, Faculty of Medicine, Universitas Indonesia, Jakarta, Indonesia \\ ${ }^{3}$ Department of Medical Acupuncture, RSUPN Dr. CiptoMangunkusumo, Jakarta, Indonesia \\ ${ }^{4}$ Universitas Negeri Jakarta, Jakarta, Indonesia
}

Received October 13, 2020

Revised January 15, 2021

Accepted January 25, 2021

Correspondence to Wahyuningsih Djaali

Medical Acupuncture Specialist Program, Faculty of Medicine, Universitas Indonesia, Jakarta, Indonesia

E-mail inchy86@yahoo.com
Background: Diabetes mellitus (DM) is a chronic metabolic disease characterized by increased blood sugar levels. The current management of DM to date has a target of controlling blood glucose, but the therapy cannot be separated from long-term drug side effects. Acupuncture can be an option as an adjunct therapy for DM.

Objectives: The purpose of this study was to compare the effectiveness between manual acupuncture and laser acupuncture.

Methods: This study was a randomized control experimental study with a pretest and posttest design using 24 male Sprague-Dawley rats divided into 4 groups: a normal group, a diabetes group, an acupuncture group, and a laser group. Manual acupuncture and laser acupuncture were performed 6 times in two weeks. Fasting blood glucose (FBG) levels, the cell density of Langerhans islets, and side effects were assessed and compared among the 4 groups.

Results: The highest mean cell density of Langerhans islets was found in the laser and acupuncture group, and the lowest was found in the diabetes group. In the post hoc analysis, the normal, acupuncture, and the laser groups had a significantly higher mean cell density than the diabetes group. The lowest mean FBG level was in the laser group, followed by the acupuncture group, and the highest was in the diabetes group, but this difference was not significant. There were no serious side effects from the use of manual acupuncture or laser acupuncture.

Conclusion: Both manual acupuncture and laser acupuncture can improve the histological findings of Langerhans islets in type 2 diabetic rats, and both are safe to use.

Keywords: EX-B3, Laser acupuncture, Manual acupuncture, Type 2 diabetes

\section{INTRODUCTION}

Diabetes mellitus (DM) is a chronic metabolic disease characterized by increased blood glucose levels. Currently, epidemiological research shows an increasing trend in the incidence and prevalence of type $2 \mathrm{DM}$ in various parts of the world. The World Health Organization (WHO) predicts an increase in the number of people with DM, which is a global health threat [1]. Half of DM cases are not diagnosed because DM is not generally accompanied by symptoms until complications occur [2]. In Indonesia, according to the results of the 2018 RISKESDAS (Riset Kesehatan Dasar), the prevalence of DM in people aged $\geq 15$ years is $8.5 \%$, which is a significant increase compared with that in $2013(6.9 \%)[3,4]$.
The management of DM to date targets controlling blood glucose, both by way of lifestyle modifications and by drug therapy, which cannot be separated from long-term drug side effects. Although many new drugs have been discovered, due to the growing population with diabetes, new effective therapies in addition to drugs are still needed. Acupuncture, which is a nonpharmacological therapeutic modality, can be an option as an adjunct therapy for DM.

Acupuncture has various stimulation methods, including manual acupuncture, electroacupuncture, thread embedding acupuncture, acupressure, laser acupuncture, and ear acupuncture, that have been used in several studies evaluating the effects and safety of acupuncture in type $2 \mathrm{DM}$. The latest systematic review in 2019 showed that studies 
on acupuncture in DM mostly use manual acupuncture modalities [1]. Selection of a manual acupuncture modality in patients with DM can increase the risk of infection in patients with uncontrolled blood glucose; thus, a laser acupuncture modality can be used as a therapeutic option.

Laser acupuncture is a therapeutic modality that uses a low-energy laser light to stimulate acupuncture points. Laser acupuncture is an innovative combination of acupuncture therapy with modern technology in the form of light with several advantages; this modality is painless, does not cause vasovagal syncope, infection, or trauma to the skin, and has a short therapy time [5].

One acupuncture point, EX-B3 Weiwanxiashu in traditional Chinese medicine, is the back Shu point of the pancreas and can reflect the physiological and pathological conditions of the pancreas. Stimulation at back Shu points is said to improve the function of the organs involved. The results of a study by Wei [6] (2004) showed that stimulation with catgut implantation at EX-B3 Weiwanxiashu can reduce fasting blood sugar levels in patients with DM. Acupuncture research on DM treatment using the single point EX-B3 Weiwanxiashu is currently not published in English.

Animal model research plays an important role in understanding the pathogenesis of diseases and particularly how acupuncture may play a role. Many animal models have been developed to determine biocellular and biomolecular mechanisms to evaluate the role of acupuncture in reversing the imbalances that occur due to disease [7]. In this study, diabetic animal models were studied to evaluate the effectiveness of manual acupuncture and laser acupuncture modalities and to compare these two modalities.

\section{MATERIALS AND METHODS}

This study was a randomized control experimental study with a pretest and posttest design. This study was conducted at Bimana Indomedical Animal Laboratory (Bogor, Indonesia) for five months March 2020 until July 2020. This research was approved by the Medical Research Ethics Committee of Faculty of Medicine, University of Indonesia, Ethics Approval No: KET/274.UN2.F1/ETIK/ PPM.00.02/2020 on March $11^{\text {th }}$, 2020.

\section{Experimental grouping}

Twenty-four Sprague-Dawley rats were divided into four groups: (1) a normal group $(\mathrm{n}=6)$; (2) a diabetes group $(\mathrm{n}=$ 6); (3) diabetic rats receiving manual acupuncture treatment, called the 'acupuncture group' ( $n=6)$; and (4) diabetic rats receiving laser acupuncture treatment, called the 'laser group' $(n=6)$. The inclusion criteria were as follows: (1) 8-10-weekold rats weighing 200-250 grams; (2) active and healthy rats (active movement and no dull hair, hair loss or balding) with a good appetite for food and drink; (3) diabetic rats (in the diabetes, acupuncture, and laser groups) with fasting blood glucose (FBG) levels $\geq 126 \mathrm{mg} / \mathrm{dL}$ after induction of DM with streptozotocin (STZ) $[8,9]$ or random blood glucose (RBG) levels > $300 \mathrm{mg} / \mathrm{dL}$ [10]; and (4) no signs of inflammation or infection at the acupuncture point location. The exclusion criteria were rats that were unable to adapt during the study.

Each rat was placed in $50 \times 50 \times 40 \mathrm{~cm}$ cage with one rat per cage until each rat's body weight reached 200 grams at a temperature of $24 \pm 1^{\circ} \mathrm{C}$ and $55 \pm 5 \%$ humidity. The lightdark cycle was performed every 12 hours (the light time was set at 8:00 AM, and the dark time was set at 10:00 PM). Drinking water was provided ad libitum. Each cage was kept clean; for normal rats, bedding replacement was performed every three days, and for diabetic rats, bedding replacement was performed every one to two days.

\section{Establishment of diabetic model}

The STZ dosage was $50 \mathrm{mg} / \mathrm{kg}$ dissolved in citric acid monohydrate with a $\mathrm{pH}$ of 4.0 (cold temperature, freshly prepared), and STZ was injected intraperitoneally to induce DM in 12 rats (Streptozotocin CAS, SangonBio Tech, Shanghai, China). FBG and RBG levels were examined ten days after STZ induction to determine the success of DM induction. Rats that showed FBG levels $\geq 126 \mathrm{mg} / \mathrm{dL}$ or RBG levels $>300 \mathrm{mg} / \mathrm{dL}$ were categorized as diabetic rats. Manual acupuncture and laser acupuncture treatment were performed one day after the rats became diabetic.

\section{Intervention}

Manual acupuncture was performed at EX-B3 Weiwanxiashu with a $0.20 \times 13 \mathrm{~mm}$ stainless steel needle (Huanqiu, China). The needle was inserted at a depth of 3-5 mm with lifting-thrusting stimulation at the beginning of the insertion; then, the needle was retained for 20 minutes. Aseptic procedure was performed prior to inserting the needle. Manual acupuncture was performed three times a week for two weeks for a total of six sessions. During the acupuncture procedure, the rats were placed on a $7 \times 7 \mathrm{~cm}$ acrylic restrainer with a height of $70 \mathrm{~cm}$ to keep the animals calm and motionless without anesthesia. EX-B3 Weiwanxiashu is located at $7 \mathrm{~mm}$ lateral to the depression under the eighth thoracic vertebral process [11].

Laser acupuncture was performed at EX-B3 Weiwanxiashu bilaterally with the following settings: wavelength of 785 $\mathrm{nm}$, continuous wave, dosage of $0.3 \mathrm{~J}$, and power of $5 \mathrm{~mW}$ for 60 seconds each point (RJ-Laser, laser pen practice ${ }^{\circledR}$ from Germany). Aseptic procedure was performed before the laser was applied. Laser acupuncture was performed three times a week for two weeks, for a total of six sessions. During the 
laser acupuncture procedure, an assistant held the rats with her hand on the head and body to keep them from moving without anesthesia. Researchers and research assistants wore special eye protection glasses due to the use of the laser light.

\section{Measurement of FBG levels}

FBG levels were measured before, after three sessions, and after six sessions of acupuncture. Measurement of FBG levels was performed at 1:00 pm after the rats had fasted for six hours. Blood was drawn through a vein at the tail end of the rat, and blood glucose levels were measured using GlucoDR.

\section{Histological examination}

The histology of the pancreas including the cell density and percentage area of Langerhans islets was analyzed using pancreas preparations that were cross-sectioned at the tail of the pancreas. The cell density of Langerhans islets in the three groups was assessed by counting the number of cells in the Langerhans islets divided by the islet area, which was observed under a microscope at $400 \times$ magnification. This calculation was performed on the three largest Langerhans islets for each preparation. All images were analyzed using ImageJ software.

\section{Side effects}

Side effects associated with acupuncture were evaluated at the end of each treatment session. We assessed for hematoma formation or infection at the acupuncture site. In the laser group, we assessed for the presence of erythematous skin.

\section{Statistical analysis}

A bivariate analysis was performed to analyze differences in the mean cell density of Langerhans islets and FBG levels of the three groups. An independent t-test was used when comparing between two groups, and one-way ANOVA was used when comparing between three groups. The significance of the test results was determined based on $p<0.05$.

\section{RESULTS}

\section{Baseline characteristics of the research subjects}

The baseline characteristics of the research subjects based on body weight and pre-induction FBG levels are shown in Table 1. The subjects' body weights and pre-induction FBG levels were not significantly different, indicating that before treatment, the four groups were equal.

\section{Descriptive profile of langerhans islets}

Histological observation of Langerhans islets revealed the following: (1) deformity of Langerhans islets, (2) irregular cell distribution, and (3) cell atrophy. Fig. 1 shown the Langerhans islets at $100 \times$ and $400 \times$ magnification in the four groups.

In the normal group, the Langerhans islets were found regularly, contained cells that were normally distributed, and had no visible cell atrophy. In the diabetes group, the shape of Langerhans islets was mostly irregular, the cell distribution was uneven, and there was considerable cell atrophy characterized by pyknotic cell nuclei. In the acupuncture and laser groups, the shape of Langerhans islets was slightly irregular, their size was quite diverse, and the cell distribution was also varied; some islets had normally distributed cells, and some were abnormal. The results of this study showed that there was a difference between normal rats and diabetic rats: in diabetic rats, there was a deformity in the Langerhans islets, and this finding was more dominant in the diabetes group than in the acupuncture and laser groups.

\section{Cell density of langerhans islets}

The cell density of Langerhans islets was assessed to evaluate the mean difference in the four groups. A bivariate analysis was performed to determine whether there was a significant difference in the mean cell density of Langerhans islets among the four groups. The results of the Shapiro-Wilk normality test showed that the data were normally distributed $(p>0.05)$, and the results of the homogeneity of variance test showed that the data were homogeneous $(p=0.291)$. Thus, the mean cell density of Langerhans islets could be compared among the four groups of rats. The graph below presents an overview of the cell density of Langerhans islets (mean and standard deviation) in the four groups.

As shown the graph (Fig. 2), the highest mean cell density of Langerhans islets was in the laser and acupuncture groups, and the lowest was in the diabetes group. The post hoc analysis showed that the normal, acupuncture, and laser

Table 1. Baseline characteristics of the research subjects

\begin{tabular}{lccccc}
\hline & \multicolumn{4}{c}{ Group } \\
\cline { 2 - 5 } \multicolumn{1}{c}{ Characteristics } & $\begin{array}{c}\text { Normal group } \\
(\mathrm{n}=6)\end{array}$ & $\begin{array}{c}\text { Diabetic group } \\
(\mathrm{n}=6)\end{array}$ & $\begin{array}{c}\text { Acupuncture group } \\
(\mathrm{n}=6)\end{array}$ & $\begin{array}{c}\text { Laser group } \\
(\mathrm{n}=6)\end{array}$ \\
\hline Body weight (grams) & $224.82 \pm 17.31$ & $234.50 \pm 17.57$ & $225.73 \pm 19.43$ & $220.13 \pm 18.78$ & 0.596 \\
FBG levels pre-induction $(\mathrm{mg} / \mathrm{dL})$ & $110.00 \pm 7.69$ & $102.67 \pm 13.49$ & $103.00 \pm 3.69$ & $97.50 \pm 9.81$ & 0.179 \\
\hline
\end{tabular}

Statistical test using one-way ANOVA. 
groups had significantly higher mean cell densities than the diabetes group.

\section{FBG levels}

FBG levels were assessed to determine the mean difference in the three groups of diabetic rats. A bivariate analysis was performed to determine whether there was a significant difference between the mean FBG levels in the three groups of diabetic rats. The results of the Shapiro-Wilk normality test showed that the data were normally distributed $(p>0.05)$, and the homogeneity of variance test results showed that the data were homogeneous $(p=0.221)$. Thus, the mean FBG

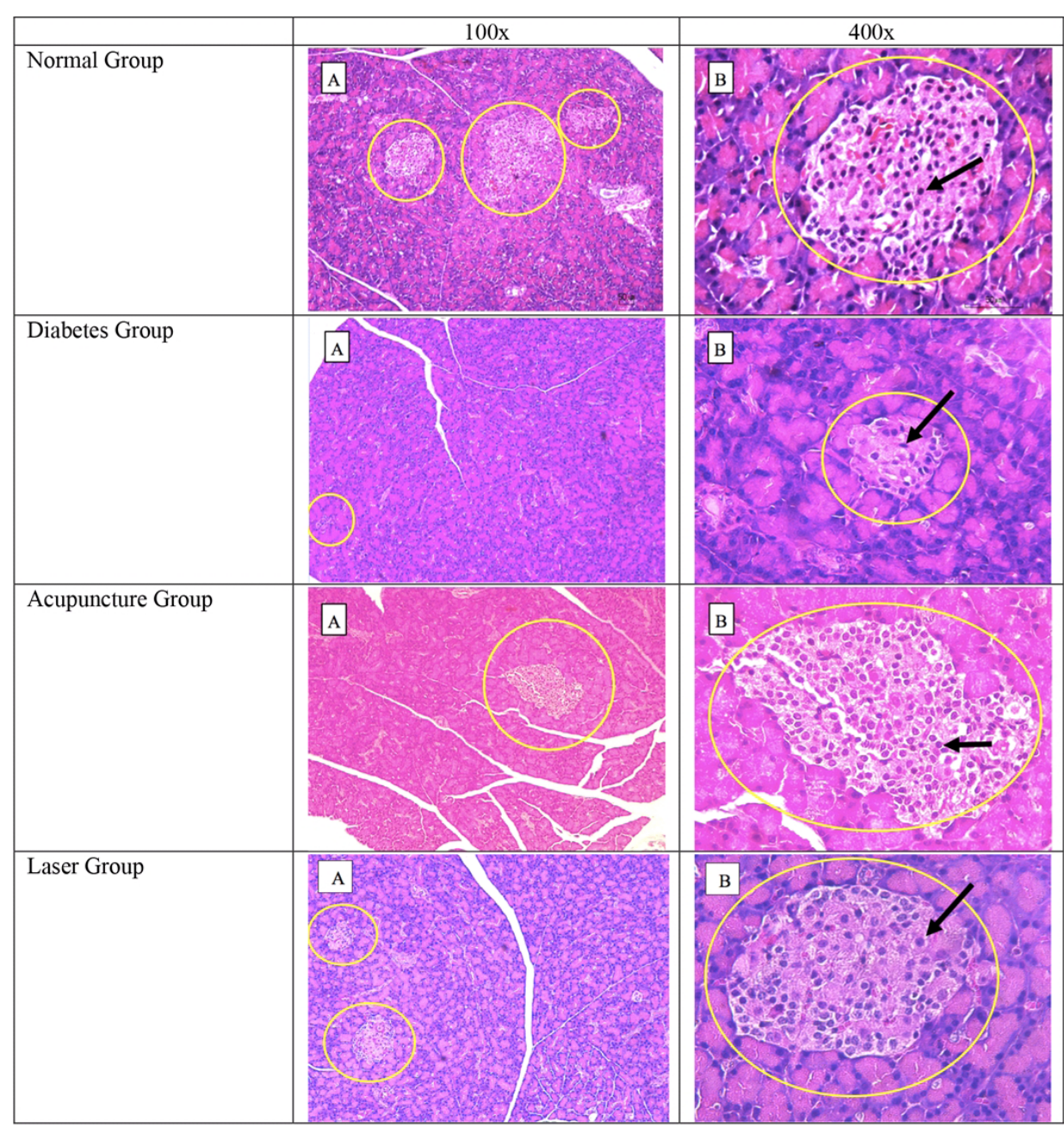

Fig. 1. Langerhans islets of the four groups. The shape of Langerhans islets is shown in yellow circles, and the cells are indicated by black arrows. Each group is displayed at $100 \times$ magnification (A) and 400x magnification (B).

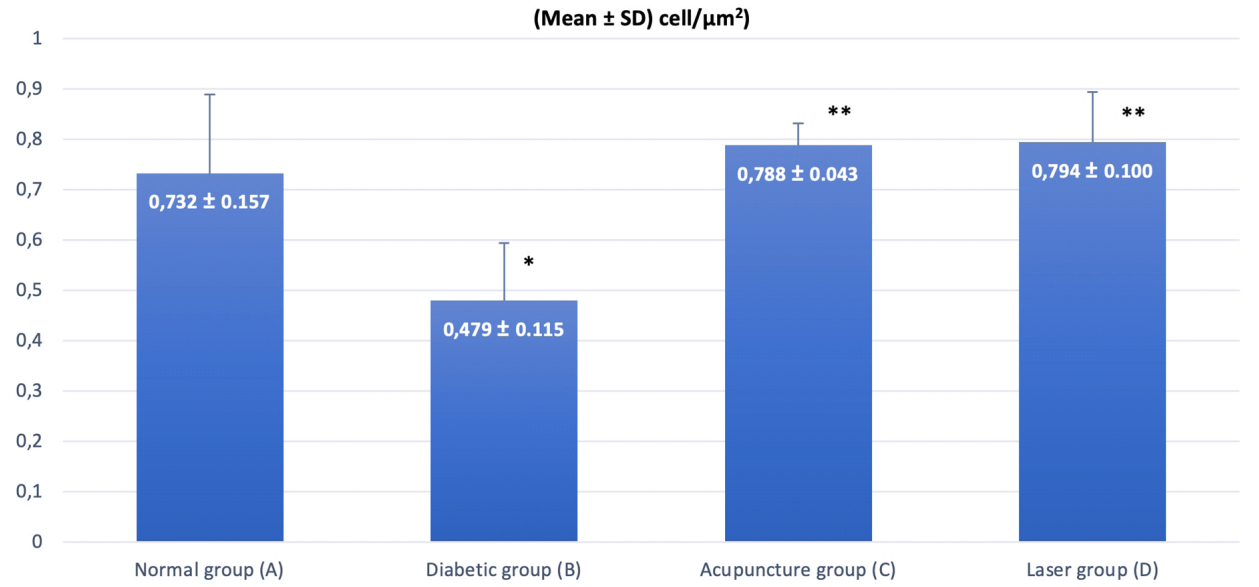

Fig. 2. Graph of the cell density of Langerhans in the four groups. ${ }^{*} p<$ 0.05 compared with the normal Group (A); ${ }^{* *} p<0.05$ compared with the diabetic Group (B). The normal (A), acupuncture (C), and laser groups (D) had significantly higher mean cell densities than the diabetes group (B). ${ }^{*} p<0.001$ with one-way ANOVA. ${ }^{*} p$ of the post hoc analysis: $A$ vs. $B=0.005 ; A$ vs. $C=$ 1.000 ; $A$ vs. $D=1.000$; $B$ vs. $C=$ $0.001 ; B$ vs. $D=0.001 ; C$ vs. $D=$ 1.000 . 


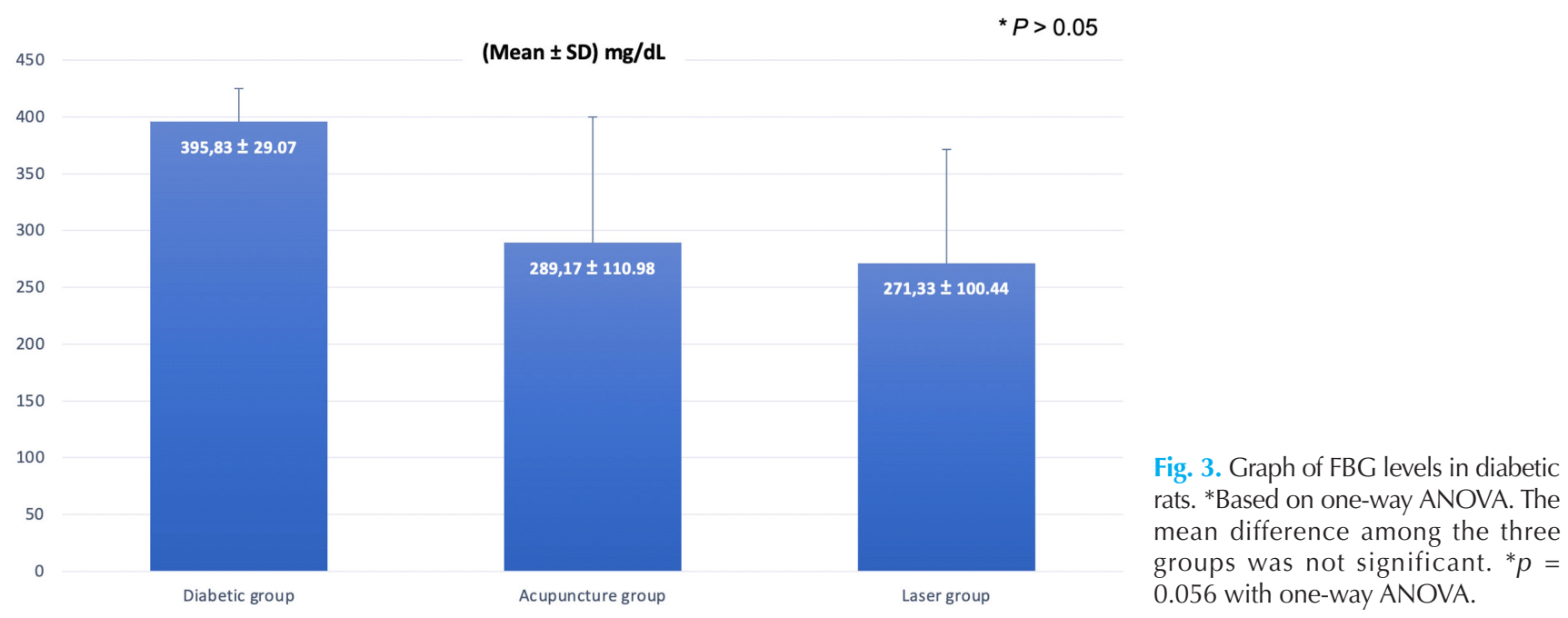

levels could be compared among the three groups. The graph below presents an overview of FBG levels (mean and standard deviation) in the three groups of diabetic rats.

As shown in the graph above (Fig. 3), the lowest mean FBG level was in the laser group, followed by the acupuncture group, and the highest was in the diabetes group, but this difference was not significant.

\section{Side effects}

There were no serious side effects from the use of manual acupuncture or laser acupuncture. There was no bleeding under the skin (hematoma formation) or infection at the puncture site in the manual acupuncture group. The only side effect was a needle extraction mark at the acupuncture point in some rats that disappeared in a few seconds. In the laser group, the visible effect was the appearance of skin erythema around EX-B3 Weiwanxiashu after laser application. Transient skin erythema can be caused due to a vasodilatory effect but will return to normal in time.

\section{DISCUSSION}

This research was conducted on 24 male Sprague-Dawley rats aged 8-10 weeks that were divided into four groups: a normal group, a diabetes group, an acupuncture group, and a laser group. The number of subjects was 6 per group, for a total of 24 rats. Of the total research subjects, none were declared null and void; thus, all subjects completed the study. Male rats were chosen for this study because they are more sensitive to STZ toxicity and are less affected by the hormone estrogen than female rats, which reduces sensitivity to STZ [12]. The body weight limit for inclusion was 200-250 grams because STZ-induced diabetic rats often experience weight loss in addition to the three typical symptoms of DM (polyuria, polydipsia, and polyphagy). Weight loss in diabetic rats occurs due to structural degradation of body-forming proteins $[12,13]$.

Based on the pancreatic histology, in the normal group, the Langerhans islets were regular in shape, had normally distributed cells, and had no visible cell atrophy. In the diabetes group, the shape of Langerhans islets was mostly irregular, the cell distribution was uneven, and cell atrophy characterized by pyknotic nuclei was observed. The results showed that there were differences in the histological findings between normal rats and diabetic rats. In diabetic rats, Langerhans islets deformities were present; this finding was more dominant in the diabetes group than in the acupuncture and laser groups, showing improved pancreatic histology, which was assessed descriptively.

The pancreatic histological preparations in this study were made with hematoxylin and eosin (HE) staining. The cells found in the Langerhans islets, including beta cells that produce insulin, were assessed with HE staining. Apart from beta cells, there are also alpha cells that produce glucagon, delta cells that produce somatostatin, $\mathrm{F}$ cells that produce pancreatic polypeptides, and epsilon cells that produce ghrelin. All of these cells have their respective proportions in one Langerhans islet. The proportion of beta cells is $65-70 \%$, that of alpha cells is $15-20 \%$, that of delta cells is $5-10 \%$, and the remaining $5 \%$ of an islet is a combination of $\mathrm{F}$ cells and epsilon cells. Therefore, the cell density of Langerhans islets represents the amount of beta cell damage that occurs due to STZ induction of DM $[14,15]$.

The histological features of the pancreas were assessed by analyzing the differences in the cell density of Langerhans islets in the four groups. The cell density was assessed by counting the number of cells in the Langerhans islets divided by the area seen under a microscope at $400 \times$ magnification. 
The highest cell density results were found in the laser group, followed by the acupuncture group. Therefore, there was improvement in histological abnormalities due to STZ induction. This effect may have been caused by beta cell multiplication through the stimulation of beta cell mitosis according to previous studies [16]. In addition, acupuncture is also known to improve and accelerate healing through stem cell mobilization [17]. The increase in the number of beta cells of Langerhans islets may have been caused by the process of cell mitosis due to acupuncture stimulation, especially laser acupuncture stimulation [16].

Laser acupuncture activates cellular metabolism in the mitochondria leading to increased adenosine triphosphate (ATP) production, modulation of reactive oxygen compounds, and induction of transcription factors. This effect increases cell proliferation and migration of cells such as fibroblasts, cytokine levels, inflammatory mediators, and tissue oxygenation; there are also secondary effects, such as anti-inflammatory effects, analgesic effects, regenerative effects, anti-edema effects, and improvement in circulation $[5,18]$. This regenerative effect is expected to play a role in the regeneration of Langerhans islets.

With regard to FBG levels, there was no statistically significant difference among the three groups of diabetic rats; however, on average, there was a decrease in FBG levels in the acupuncture and laser groups. The results of this study are consistent with those of a study by Jiang et al. [13] (2011), where one of the objectives of the study was to determine the therapeutic effect of acupuncture in diabetic rats due to STZ induction. In the results of that study, blood glucose levels in rats in the acupuncture group were significantly lower than those in rats in the diabetes group. The study also concluded that acupuncture could significantly reduce hyperglycemic conditions, insulin deficiency, weight loss, and damage to the Langerhans islets in diabetic rats. Another study by Nakamura et al. [19] (2014) examined the effects of acupuncture therapy on Otsuka Long-Evans Tokushima Fatty (OLETF) rats, which are rats with type 2 DM. The results showed that blood glucose levels in the DM group treated with acupuncture were significantly lower than those in the DM group that was not treated, and the blood glucose levels in the group of normal rats were significantly lower than those in the two groups of DM rats. Both studies used manual acupuncture as a therapeutic modality.

A study by Cornejo-Garrido et al. [20] (2014) used laser acupuncture at BL20 Pishu in STZ-induced type 1 DM rats to determine the effect of reducing blood glucose levels. The results showed that blood glucose levels in the DM group treated with laser acupuncture were significantly lower after 28 days of treatment than those the control group $(p<$ $0.05)$. In this study, there were no side effects related to blood biochemical profile changes or blood cell counts due to laser acupuncture, and there was no internal organ damage due to laser use.

The results of this study indicate that acupuncture can reduce FBG levels in DM rats as well as improve pancreatic histology, which indicates improved pancreatic function. Thus, because the effectiveness of acupuncture was observed with very minimal side effects, acupuncture can be applied to DM patients.

\section{CONCLUSIONS}

Both manual acupuncture and laser acupuncture can improve the histological findings of Langerhans islets in type 2 diabetic rats. The morphology and cell density of Langerhans islets showed improved results with laser acupuncture. Both manual acupuncture and laser acupuncture are safe to use with minimal side effects.

\section{ACKNOWLEDGEMENTS}

The authors thank Dwi Rachma Helianthi as the head of the Medical Acupuncture Specialist Program in Faculty of Medicine, Universitas Indonesia for her invaluable advice and support during this research. The authors would also like to thank Bimana Indomedical, Bogor, Indonesia, for providing an animal laboratory and the Department of Histology, Faculty of Medicine, Universitas Gunadarma, Indonesia, for the assistance in histological analysis. This work was funded by a grant from Universitas Indonesia (Grant no: NKB-910/ UN2.RST/HKP.05.00/2020) "Hibah Publikasi Terindeks Internasional (PUTI) Prosiding 2020".

\section{CONFLICT OF INTEREST}

The authors declare no conflict of interest.

\section{ORCID}

Ahmad Aulia Jusuf,

https://orcid.org/0000-0002-1061-5813

Yoshua Viventius, https://orcid.org/0000-0002-4846-2373 Wahyuningsih Djaali,

https://orcid.org/0000-0002-7101-0228

\section{REFERENCES}

1. Chen C, Liu J, Sun M, Liu W, Han J, Wang H. Acupuncture for type 2 diabetes mellitus: a systematic review and meta-analysis of randomized controlled trials. Complement Ther Clin Pract 2019;36:100-12. 
2. Saputra NT, Suartha IN, Dharmayudha AAGO. [Diabetagonik agent streptozocin to make white rats male diabetes mellitus]. Bul Vet Udayana 2018:10;116-21. Indonesian.

3. Badan Penelitian dan Pengembangan Kesehatan. Hasil Utama Riskesdas 2018. Available at: https://kesmas.kemkes.go.id/ assets/upload/dir_519d41d8cd98f00/files/Hasil-riskesdas2018_1274.pdf [Date accessed: February 2, 2020] Indonesian.

4. Soelistijo SA, Novida H, Rudijanto A, Soewondo P, Suastika K, Manaf A, et al. Konsensus Pengelolaan dan Pencegahan Diabetes Melitus Tipe 2 Di Indonesia 2015. Jakarta: PB PERKENI, 2015. Indonesian.

5. Kreisel V, Weber M. A Practical Handbook: Laser Acupuncture Successful Treatment Concepts. Starnberg: Füchtenbusch, 2012.

6. Wei D. Catgut implantation at Weiwanxiashu (Ex-B 3) for diabetes mellitus. J Acupunct Tuina Sci 2004;2:15-7.

7. Al-Awar A, Kupai K, Veszelka M, Szűcs G, Attieh Z, Murlasits Z, et al. Experimental diabetes mellitus in different animal models. J Diabetes Res 2016;2016:9051426.

8. Firdaus F, Rimbawan R, Marliyati SA, Roosita K. [Streptozotocin, sucrose- induce diabetic male rats model for research approach of gestational diabetes mellitus]. Media Kesehat Masy Indones 2016;12:29-34. Indonesian.

9. Jung JY, Lim Y, Moon MS, Kim JY, Kwon O. Onion peel extracts ameliorate hyperglycemia and insulin resistance in high fat diet/ streptozotocin-induced diabetic rats. Nutr Metab (Lond) 2011;8: 18.

10. Wilson RD, Islam MS. Fructose-fed streptozotocin-injected rat: an alternative model for type 2 diabetes. Pharmacol Rep 2012; 64:129-39.

11. Cao BY, Li R, Tian HH, Ma YJ, Hu XG, Jia N, et al. PI3KGLUT4 signal pathway associated with effects of EX-B3 electro- acupuncture on hyperglycemia and insulin resistance of T2DM rats. Evid Based Complement Alternat Med 2016;2016:7914387.

12. Furman BL. Streptozotocin-induced diabetic models in mice and rats. Curr Protoc Pharmacol 2015;70:5.47.1-5.47.20.

13. Jiang YL, Ning Y, Liu YY, Wang Y, Zhang Z, Yin LM, et al. Effects of preventive acupuncture on streptozotocin-induced hyperglycemia in rats. J Endocrinol Invest 2011;34:e355-61.

14. Ovalle WK, Nahirney PC. Netter's Essential Histology, 2nd ed. London: Elsevier Health Sciences, 2013.

15. Young B, Woodford P, O'Dowd G. Wheater's Functional Histology: A Text and Colour Atlas, 6th ed. London: Elsevier Health Sciences, 2013.

16. Abdurachman H, Suhariningsih S, Rubiyanto A. Laser acupuncture at BL20 Point Stimulate Pancreatic Beta cell in type 1 diabetes mellitus. Bali Med J 2019;8:241-6.

17. Gu G, Brown JR, Melton DA. Direct lineage tracing reveals the ontogeny of pancreatic cell fates during mouse embryogenesis. Mech Dev 2003;120:35-43.

18. Hamblin MR, Agrawal T, de Sousa M. Handbook of Low-Level Laser Therapy. Singapore: CRC Press, 2016.

19. Nakamura H, Ishigami T, Kawase Y, Yamada A, Minagawa M, Fukuta $\mathrm{H}$, et al. Effects of acupuncture stimulation on blood glucose concentration in the Otsuka Long-Evans Tokushima Fatty (OLETF) rat, an animal model for type-2 diabetes mellitus. Med Sci Monit Basic Res 2014;20:70-5.

20. Cornejo-Garrido J, Becerril-Chávez F, Carlín-Vargas G, Ordoñez-Rodríguez JM, Abrajan-González Mdel C, de la CruzRamírez R, et al. Antihyperglycaemic effect of laser acupuncture treatment at BL20 in diabetic rats. Acupunct Med 2014;32:48694. 